\title{
CIM como integração de dados municipais: uma possibilidade para o planejamento urbano \\ $\mathrm{CIM}$ as integration of municipal data: a possibility for urban planning
}

\author{
Júlio Cesar dos Santos \\ Universidade Federal de Pernambuco | Recife - PE| Brasil | julio.csantos2@ufpe.br \\ Max Lira Veras Xavier de Andrade \\ Universidade Federal de Pernambuco | Recife - PE | Brasil | max.andrade@ufpe.br
}

\section{Resumo}

As Tecnologias da Informação e Comunicação vem se tornando um meio de auxiliar na promoção de um planejamento urbano mais efetivo. Nesse contexto, a modelagem da informações da cidade emerge como uma forma eficiente de planejamento urbano. Este trabalho explora o conceito de City Information Modeling - Data Layer (CIM-DL) como proposta para o delineamento de um sistema de auxílio ao processo decisório de planejamento urbano. Esse trabalho apresenta os resultados preliminares de uma pesquisa de mestrado que faz uso do Design Science Research para propor um modelo de CIM-DL. Os resultados parciais apontam para a necessidade da concepção de um protocolo estruturado para gestão da informação das cidades, que sirva como guia para a criação de um sistema integrador de dados a ser usado para auxiliar o processo decisório do planejamento e gestão dos municípios brasileiros.

Palavras-chave: Modelagem de Informações da cidade. Sistema de Informações Municipais. Cruzamento de dados. Tecnologias de Informação e Comunicação.

\begin{abstract}
Information and Communication Technologies are becoming a way of promoting more effective urban planning. In this context, city information modeling emerges as an efficient form of urban planning. This work explores the concept of City Information Modeling - Data Layer (CIM-DL) as a proposal for the design of a system to aid the decision-making process of urban planning. This work presents the preliminary results of a master's research that uses Design Science Research to propose a CIM-DL model. The partial results point to the need to design a structured protocol for information management in cities, which will serve as a guide for the creation of an integrative data system to be used to assist in the decision-making process of planning and management in Brazilian municipalities.
\end{abstract}

Keywords: CIM - City Information Modeling. Municipal Information System. Data crossing. Information and Communication Technologies.

\section{INTRODUÇÃO}

Até o ano de 2050 estima-se que a população mundial alcance o número de 9,7 bilhões de pessoas, um acréscimo de 2 bilhões em relação ao número atual, esse aumento se

Como citar:

SANTOS , J. C. dos; ANDRADE , M. L. V. X. de. CIM como integração de dados municipais: uma possibilidade para o planejamento urbano. In: SIMPÓSIO BRASILEIRO DE TECNOLOGIA DA INFORMAÇÃO E COMUNICAÇÃO NA CONSTRUÇÃO, 3., 2021, Uberlândia. Anais [...]. Porto Alegre: ANTAC, 2021. p. 1-7. Disponível em: https://eventos.antac.org.br/index.php/sbtic/article/view/620. Acesso em: 3 ago. 2021.p.1-11. 
dará essencialmente nas cidades que já existem, aumentando a pressão sobre o meio ambiente e os sistemas urbanos [1]. Portanto, devido ao crescimento das cidades não possuir indicativos de desaceleração ou retorno, emerge a necessidade de uma urbanização mais eficiente e sustentável, a qual vem se alinhando com aplicações de Tecnologias da Informação e Comunicação - TIC como maneira de otimizar recursos enquanto diminui custos. Os avanços nas TIC promovem uma metamorfose nas cidade, alterando sua dinâmica e se alinhando a sistemas digitais fortemente baseados na coleta, tratamento e uso de dados. Essa dinâmica digital tem se mostrado alinhada com novas problemáticas, em que esse novo mundo dos dados pode auxiliar a tornar as cidades mais limpas, seguras e funcionais por meio do uso de sensores, dispositivos responsivos e microcomputadores capazes de se comunicar pela internet [2].

A compreensão do espaço, a partir de bancos de dados precisos de informação da cidade e da modelagem digital de ambientes urbanos, pode clarificar o diagnóstico do ambiente urbano, bem como, das possíveis soluções para os problemas diagnosticados. Assim, torna-se possível se chegar a soluções mais rápidas e assertivas, auxiliando a tomada de decisão de planejamento das cidades. O planejador, ao ter em mãos informações precisas e atualizadas da dinâmica urbana das cidades, tem maior probabilidade de acerto nas estratégias e ações em busca da resolução dos problemas considerados importantes. Desta forma:

faz-se necessária, portanto, uma abordagem sistêmica voltada ao estabelecimento de um modelo de informação da cidade, constituído com a finalidade de ordenar e permitir uma maior interoperabilidade entre estas soluções, aumentando coeficientes de compatibilidade, gerando dados mais uniformes e permitindo tomadas de decisão melhor fundamentadas [3]

Embora um tratamento estruturado e global dos diversos sistemas que gerenciam a informação da cidade permita uma maior efetividade nas mudanças urbanas, a própria estrutura federativa brasileira é moldada na construção de instituições que estabeleçam interdependência entre os níveis de governo com o intuito de criar um controle mútuo entre os pactuantes [4]. Os municípios brasileiros, pelo seu aparato administrativo guiado na distribuição das responsabilidades urbanas por secretarias, acabam também por formar ilhas administrativas, cada uma com dados próprios ao seu domínio. Essas ilhas formam um verdadeiro arquipélago de dados não conectados, deixando submerso um potencial latente para a gestão e o planejamento urbano, entre tantas outras aplicações.

Essa composição de serviços pulverizados adiciona complexidade ao trabalho dos planejadores e gestores de modo que, mesmo a elaboração de um diagnóstico urbano simples, se torna uma tarefa exigente para a coleta de dados. Esse espaço virtual construído por meio das TIC permite abordar novas questões relacionadas às novas formas de produzir e consumir o espaço [5] e, portanto, também surgem novas formas de visualizar e planejar o espaço como o City Information Modeling - CIM (ou modelagem da informação da cidade).

Para tanto, e diante dos fatores que acarretam o aumento das complexidades da vida contemporânea, também aumenta a importância do aperfeiçoamento de métodos e técnicas para gerir esse tecido, composto de elementos estáticos e dinâmicos, 
organizados e sobrepostos naquilo que se conhece por espaço urbano. Tal processo tem sido reforçado e condicionado de maneira contínua pelas TIC [6]. Desta forma, e ainda em vistas ao aumento da pressão nos sistemas urbanos pela crescente populacional, se faz necessária a modelagem de um CIM adequada a realidade brasileira e, sobretudo, focada no ambiente público.

Este trabalho se debruça sobre a proposição de um modelo de integração de dados municipais baseado no conceito de CIM por arranjo de dados, produzindo um artefato denominado aqui como cidade SABIDA (Sistema de Armazenamento Baseado em Informações, Dados e Análises da cidade) que viabilize esta integração de maneira alinhada com a divisão político-administrativa dos municípios brasileiros. A relevância do tema é dada tanto pela caracterização intrínseca do urbanismo como área de pesquisa transdisciplinar, quanto pela crescente complexidade do ambiente urbano, de tal forma que a integração de dados entre as ilhas administrativas que compõe a gestão das cidades seja facilitada e otimizada para o oferecimento de serviços melhores e mais eficientes, de uma tomada de decisão mais célere e assertiva, facilitando o processo decisório e o planejamento urbano.

\section{PERCURSO METODOLÓGICO}

Este trabalho representa parte de uma pesquisa de mestrado que utiliza como método o Design Science Research - DSR. O DSR é um método de pesquisa composto de sub etapas que permite ao pesquisador gerar produtos visando alcançar os objetivos propostos. Trata-se, portanto, segundo Oliveira [7], de um método que possui como característica principal a orientação à solução de problemas específicos, não necessariamente buscando a solução ótima, mas a satisfatória para a situação.

O esquema de condução de uma pesquisa baseada em DSR pode ser observado na Figura 1, em que Lacerda et al [8] realizaram a divisão das etapas da pesquisa de acordo com diversos autores em que, em síntese, o fluxo do conhecimento vai da conscientização do problema até a sua conclusão, passando por um formalismo lógico de abdução ou dedução, esperando como saída desse procedimento algum constructo que resolva o problema inicial, e, por fim, esse artefato recebe uma nova avaliação a fim de identificar em qual nível este soluciona o problema inicial, fechando o ciclo da DSR. 
Figura 1 :Esquema de condução do Design Science Research (TAKEDA et al., 1990; VAISHNAVI; KUECHLER, 2009; MANSON, 2006; PEFFERS et al., 2008)
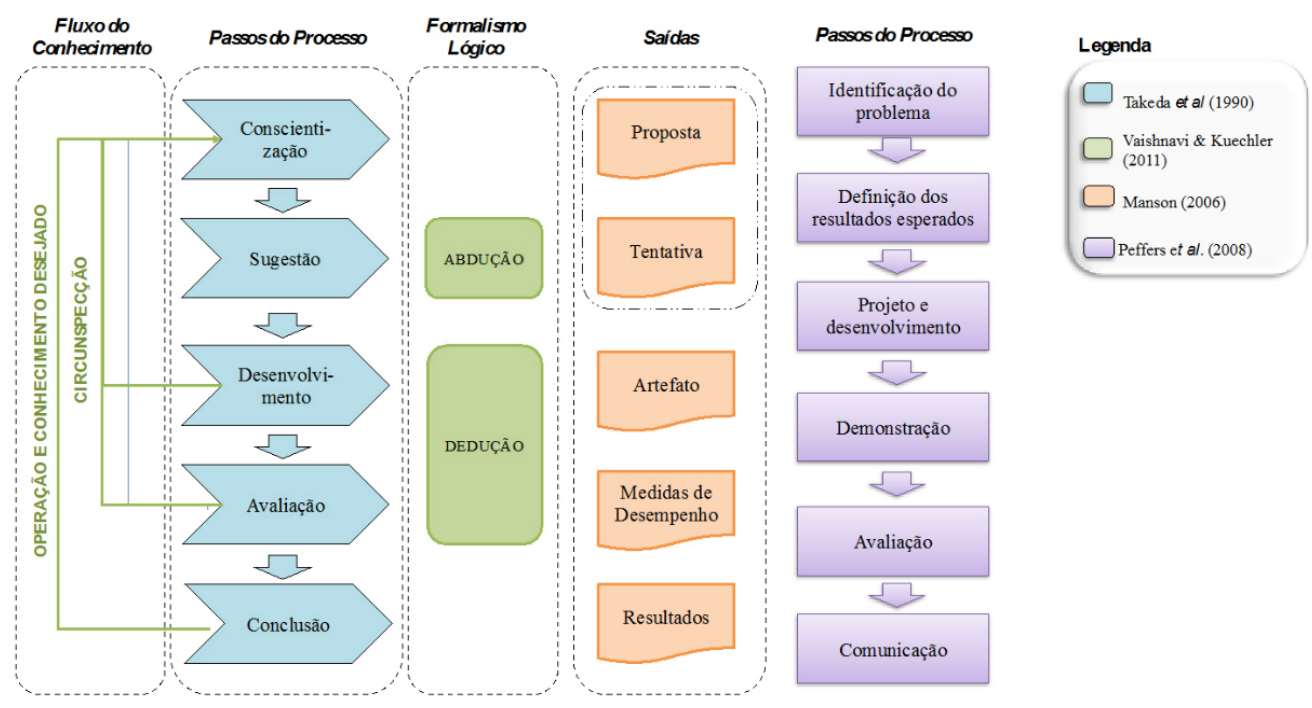

Fonte: (LACERDA et al., 2013)

A partir do esquema representado na Figura 1, foi elaborada uma adaptação deste processo em sua equivalência ao que se espera desta pesquisa (Figura 2), ou seja, apresentando a cada passo do processo metodológico sua respectiva atribuição, sendo este artigo a apresentação da etapa de desenvolvimento da modelagem conceitual do artefato.

Figura 2 :Esquema de condução do Design Science Research adaptado
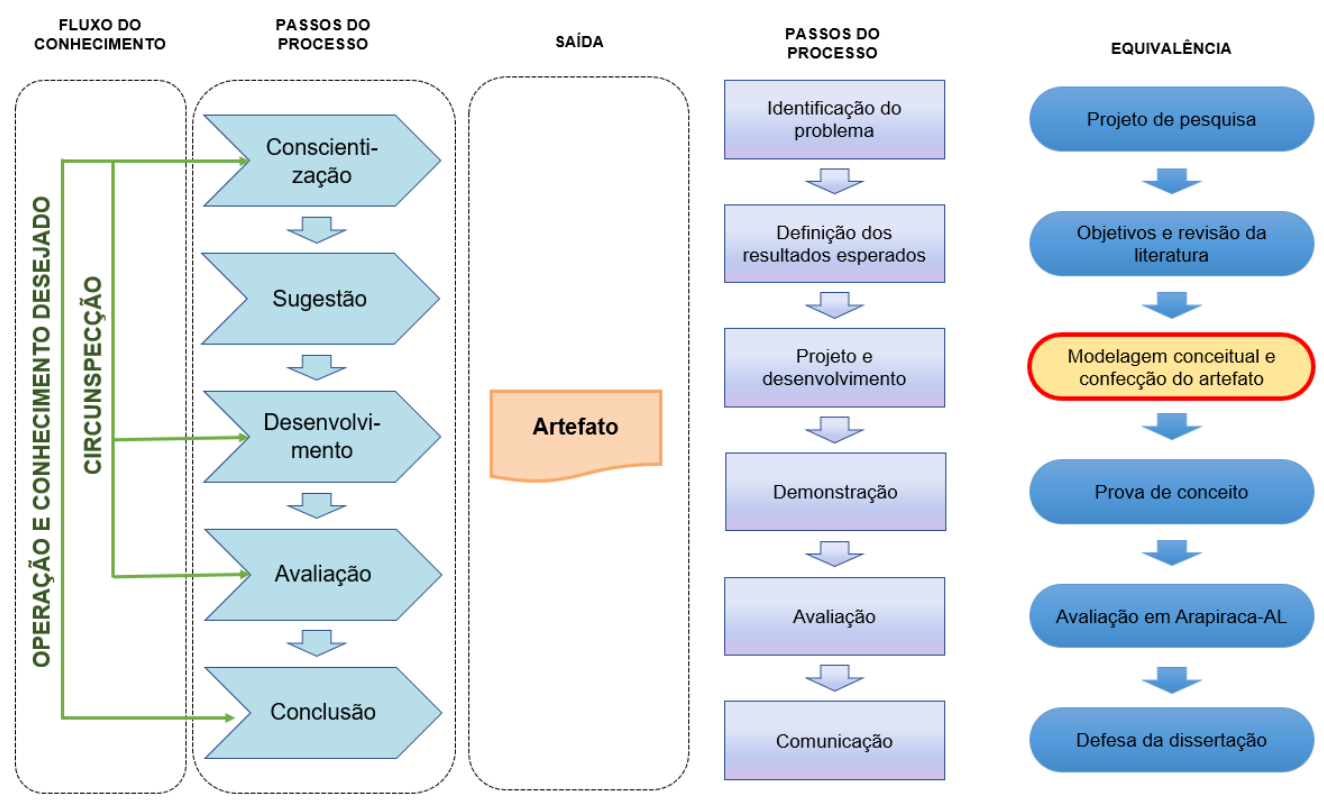

Fonte: os autores.

O artigo está estruturado em mais 4 tópicos além da introdução e do percurso metodológico, sendo eles a discussão do CIM como arranjo de dados, do Cadastro Multifinalitário como encontro de dados, até as potencialidades do sistema Cidade SABIDA, finalizando com a descrição e considerações acerca da modelagem do artefato. 


\section{CIM COMO ARRANJO DE DADOS}

A modelagem de dados da cidade tem sido alvo de pesquisa há anos, e por não haver consenso entre a delimitação de seu conceito, existem diversas aderências a outros temas, embora o entendimento mais comum seja que o CIM surge do aprofundamento de estudos a respeito da modelagem e georreferenciamento da informação (BIM e GIS) a partir dos anos 2000 [6]. O CIM, enquanto modelagem da informação, tem potencialidades que vão muito além da mera representação gráfica. O cruzamento da informação obtida de diversas fontes da cidade aparece como uma maneira eficiente de utilizar estas mesmas informações em favor do planejamento e da gestão urbana.

O CIM é ainda uma área de pesquisa emergente e um conceito ligado mais fortemente a academia, envolvendo diversas áreas de pesquisa ligadas ao urbanismo, tais como a geografia, cartografia, estatística, engenharias e ciências da computação [3]. O entendimento mais comum a respeito deste conceito trata o CIM como uma união entre os conceitos de Building Information Modeling - BIM e Geographic Information System - GIS. Contudo, ao realizar uma abordagem ontológica sobre CIM, Almeida [3] investiga as diversas aproximações de CIM com outras variantes conceituais, estabelecendo categorias diante dos preceitos atuais.

Almeida [3] conceitua 3 categorias que emergem dos trabalhos publicados a respeito de CIM, sendo duas com uma maior ênfase nos aspectos sintáticos e físicos (CIM por Ambiente Construído - CIM-BE ${ }^{1}$, e CIM por parâmetros da forma - CIM-SP²) quando tratam da modelagem da informação da cidade, e uma terceira mais abstrata que se aproxima dos aspectos semânticos dos dados da cidade e de seu arranjo qualitativo, o CIM por camada de dados ou CIM-DL ${ }^{3}$, que segundo o mesmo autor:

É uma abordagem mais fenomenológica de CIM, a qual observa a cidade sob a ótica dos eventos presentes e passados com o propósito de diagnosticar e prever determinadas situações. Corresponde à faceta de CIM mais aproximada à gestão de políticas públicas, a qual lida com tomadas de decisão fundadas em relações multidisciplinares e cuja territorialização por meio da representação digital contribui para uma compreensão mais específica de cada situação. ([3] p.73. grifo nosso)

Almeida [3] também expõe que o principal desafio dessa abordagem está na modelagem e manutenção da integração dos dados intersetoriais mantendo-os atualizados e podendo repercutir nos processos de criação desses dados, porém, como resultado, esse modelo representa dados ou conjuntos de dados distribuídos geograficamente no território, tornando a representação tridimensional desejável, mas não obrigatória.

\footnotetext{
${ }^{1}$ BE como acrônimo de Built Environment (Ambiente Construído), nota de Almeida (2018)

${ }^{2}$ SP como acrônimo de Shape Parameters (Parâmetros da Forma), nota de Almeida (2018)

${ }^{3}$ DL como acrônimo de Data Layers (Camadas de Dados), nota de Almeida (2018)
} 


\section{CTM, O TERRITÓRIO COMO ENCONTRO DE DADOS}

Considerando que as fontes de dados para o planejamento urbano são diversas, de imagens orbitais de alta resolução até dados de instituições e autarquias, além de pesquisas como as realizadas pelo IBGE, existe ainda o acervo massivo de dados do Cadastro Territorial Multifinalitário - CTM [9]. O CTM consiste no inventário territorial oficial e sistemático do município ${ }^{4}$, tratando-se de um sistema de registros espaciais que representam a estrutura urbana composto por um elemento espacial (geometria) e outra descritiva. A base de dados para o aprimoramento das cidades que as prefeituras possuem ainda conta também com um largo acervo de dados oriundos de diversos processos (tais como diagnósticos para intervenção urbana, Plano Diretor, Estudos de Impacto Ambiental, licenciamentos, etc.). Portanto, pode-se observar que atualmente a maior parte das prefeituras brasileiras possui enormes repositórios de dados potenciais para o aprimoramento da gestão e da vida urbana [10].

O CTM, com seu aspecto de inventário oficial e sistemático da cidade, em um modelo de integração de dados espaciais e descritivos entrelaçado com o conceito CIM-DL criaria um acervo de possibilidades para o planejamento urbano e promoção da cidadania limitados apenas pela criatividade do urbanista, sendo esse na verdade uma nova espécie de desafio contemporâneo, como explicado por Thompson et al, citado por Almeida:

(...) o desafio dos urbanistas contemporâneos não está mais (...) na coleta e geração de dados atualizados, mas em como relacioná-los de modo a integrar um montante cada vez mais massivo de informações com a finalidade de servir mais eficientemente ao planejamento e governança das cidades. [3].

Uma abordagem um pouco mais arrojada seria capaz de liberar o potencial de uma nova agenda para as cidades de uma forma integralizadora e socialmente mais justa. Uma cidade que se mostrasse inteligente para todos e não apenas para os cidadãos da primeira fila ${ }^{5}$. Neste trabalho, essa visão é consolidada na proposta de um sistema que integre as visões de modelagem da informação com um aparato já existente nos municípios brasileiros.

Os benefícios do arranjo de dados através de tecnologias urbanas na gestão das cidades aparecem na bibliografia pesquisada de maneiras diversas e em aplicações com enfoque na otimização de aspectos tais como o trânsito, a infraestrutura urbana, a sustentabilidade, a saúde, dentre outros, conformando uma espécie de sistema operacional urbano que integra uma nuvem de sistemas em um ponto nodal que realiza uma forma de coreografia urbana através dos dados. Embora a ideia de Sistema Operacional Urbano receba críticas por seu estilo altamente tecnocrático [11], sua aproximação aos preceitos participativos é um desafio que pode trazer benefícios concretos no que se refere a otimização da integração de dados urbanos.

\footnotetext{
${ }^{4}$ De acordo com artigo primeiro da Portaria 511/2009 do Ministério das Cidades

${ }^{5}$ Referência ao livro Confiança e medo na cidade (BAUMAN, 2009) onde o autor classifica uma espécie de polarização e estranhamento social entre ricos e pobres, incluídos e excluídos, entre o cidadão da primeira fila e da última fila.
} 


\section{POTENCIALIDADES DO CIDADE SABIDA}

Atualmente, as cidades têm a sua forma definida pela incorporação de sistemas informacionais de controle. Estas tecnologias invisíveis controlam os sistemas de abastecimento, os fluxos de trânsito e energia. Neste contexto a cidade deixa de ser um sistema estático, constituída apenas pelas suas componentes físicas, e passa a ser um "organismo vivo" [12].

O processo de Revisão Sistemática da Literatura desta pesquisa está compilado em um artigo enviado a um periódico nacional, em que a busca por sistemas de informação que gerenciem aspectos urbanos nos repositórios de trabalhos acadêmicos retornou diversos trabalhos que relatavam aplicações práticas destes sistemas nas mais diversas áreas. Os principais benefícios estão ligados a área de transporte, infraestrutura e informatização, contudo também existem aplicações para saúde, educação, meio ambiente e o próprio cadastro fiscal. Grosso modo, as aplicações e sistemas que emergem destes casos sempre objetivam utilizar os dados existentes e gerados em um modelo de organização que incremente a eficiência dos processos já existentes, conformando um Sistema de Informações Territoriais.

\section{APLICAÇÕES NO AMBIENTE URBANO}

Autili et al. [13], ao estudarem o desenvolvimento na cidade de Áquila na Itália, apresentam um estudo de caso sobre o Sistema de Mobilidade Rodoviária Colaborativa (Collaborative Road Mobility System - CRMS) que permite que veículos e infraestrutura de transporte se interconectem, compartilhando dados para coordenar suas ações para serviços como um aplicativo móvel que auxilia motoristas na experiência de uma direção mais ecologicamente correta. Outros autores argumentam que uma modelagem integradora entre bancos de dados geográficos para trânsito [14] aliadas a uma plataforma de análise visual [15] permitem superar a lacuna criada pelos vários sistemas independentes que as cidades utilizam para gerenciar o trânsito, grosso modo, criando uma plataforma e metodologia que agreguem dados de múltiplos formatos em múltiplas fontes, inclusive fontes de dados abertos [16]. Tais canais de integração são complexos e exigem a harmonização dos dados por diversos meios, seja na categorização por camadas (especialmente quando dados geográficos), seja pela realização de consultas que eliminem redundâncias, combinem fatores e permitam ao final do processo uma contribuição efetiva para a gestão do trânsito. Também existem aspectos ligados a informatização das cidades onde a visão da união de sistemas se adere a várias análises, tais como análises de redes sociais e o compartilhamento de informações entre os setores público e privado. Esses dados estão distribuídos em sistemas proprietários e abertos, o que demanda a compilação de padrões, harmonização de dados e serviços de interoperabilidade para armazenamento e processamento em vias de responder questões políticas e cientificas [17]. Souza et al. [18] utilizaram técnicas de Big Data, aprendizagem de máquina e processamento de linguagem natural para processar 7,5 milhões de postagens no Twitter, georreferenciando 286.000 destas no período da Copa do Mundo FIFA 2014. Como resultado, dentre outros, conseguiram traçar um perfil de demanda turística da cidade de Natal - RN, identificando 25 nacionalidades, além de 
uma análise sentimental no conteúdo publicado, como postagens positivas, neutras e negativas. Ainda existem soluções vinculadas a criação de um ecossistema de informações público privadas [19], visualização 3D do cadastro fiscal a partir de tecnologias BIM [20], e criação de indicadores para o desenvolvimento sustentável [21].

\section{DESCRIÇÃO DO CIDADE SABIDA}

A visão global do SABIDA busca uma união facilitada para entrada e saída de dados vinculados a diversos fins, que vão desde o monitoramento da cidade até o incentivo criativo para a composição de serviços diversos. Portanto, este sistema deve possuir também uma metodologia que contemple a entrada de dados no sistema, seu armazenamento e catalogação para consulta, além de sua saída e distribuição para outros sistemas.

A ideia central do cidade SABIDA é de criar um ponto nodal onde os dados possam ser cruzados e analisados de maneira lateral, ou seja, cruzando variáveis de aspectos distintos para permitir a criação de conhecimento, a informação contextualizada. Para isso o subsistema tem que ser modelado com uma arquitetura aberta e atualizável, que permita a entrada de dados de fontes variadas e diversas ao longo do tempo, tais como dados do recenseamento (10 anos), do organograma municipal (4 anos), do plano plurianual ( 2 anos) e das escolas (anuais ou mesmo diárias da frequência escolar), atendimentos médicos, licenças e projetos aprovados pelo município, ou mesmo informações em tempo real do trânsito, clima, acidentes, etc.

As aplicações possíveis são as mais diversas, se pode pensar em uma "WikiUrbana" onde os cidadãos podem depositar informações da cidade, tais como a história de bairros, eventos regionais e cultura local, a abertura de plebiscitos participativos pela internet, ou mesmo uma biblioteca digital municipal. Também é fácil vislumbrar a composição de cadastros temáticos realizados pela própria população em um modelo gameficado, de maneira que os cidadãos ganhem pontos e recompensas para a realização de um cadastro arbóreo por exemplo, ou até denuncias para ligações clandestinas de água e energia. Um modelo esquemático pode ser visualizado na Figura .

Figura 3: Modelo esquemático Cidade SABIDA

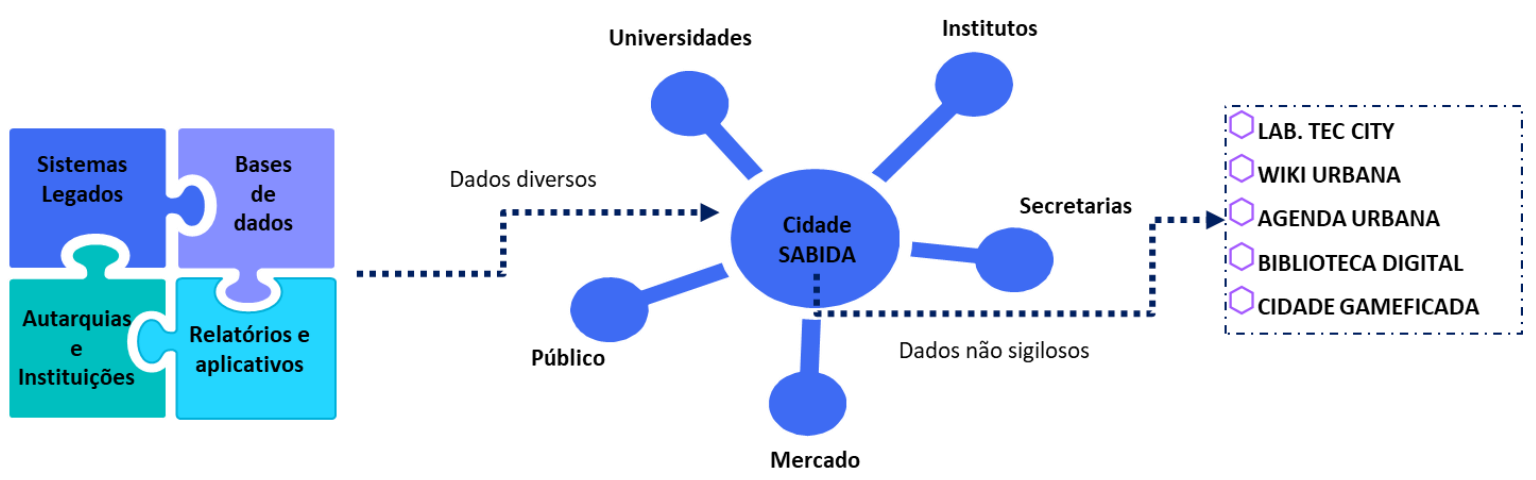

Fonte: os autores. 
A partir da perspectiva apresentada é fácil deduzir que os debates sobre o planejamento urbano estão hoje orientados à utilização de bases de dados amplas, e ainda que os impactos possíveis e as barreiras teóricas desse sistema são cingidas apenas pela criatividade dos planejadores. Se pode tratar de um leque amplo que aborde um processo decisório mais assertivo e atenda demandas de maior complexidade, demandas estas que atualmente já possuem relações imbricadas, como no caso da sustentabilidade, em que a aceleração das transformações climáticas exige respostas tão complexas quanto o próprio tema e ainda mais rápidas que seus impactos.

Os resultados parciais encontrados nesse artigo apontam que a elaboração de um sistema que atue como ponto de encontro de dados para a modelagem de um CIM-DL necessita de um protocolo mais complexo do que o abordado em outros trabalhos. $\mathrm{A}$ proposta deste artigo, mesmo que parcial, foi de apresenta um modelo conceitual de sistema de integração de dados municipais para auxiliar no processo decisório do planejamento e gestão urbana. É importante considerar que esse modelo, ainda em desenvolvimento, será implementado em uma ferramenta computacional e testado com a modelagem de uma prova de conceito, ainda a ser desenvolvido.

Por fim, é importante considerar que os resultados desta pesquisa apontaram para uma modelagem de um sistema que tenha um formato de dados aberta, de modo que seja adaptável aos diversos sistemas e variedades de dados que estão disponíveis para o planejamento urbano, sejam estes dados de levantamentos, sistemas legados, sensores ou até mesmo mídias sociais. Embora as perspectivas de utilização do sistema sejam aparentemente pouco limitadas, este artigo aborda apenas um recorte da pesquisa. Por ora, a proposta de Cidade SABIDA, aqui apresentada, não apresenta tanta sabedoria, ainda.

\section{AGRADECIMENTOS}

O presente trabalho foi realizado com apoio da Coordenação de Aperfeiçoamento de Pessoal de Nível Superior - Brasil (CAPES) - Código de Financiamento 001.

\section{REFERÊNCIAS}

[1] NAÇÕES UNIDAS. Relatório: World Urbanization Prospects 2018. 2018. Disponível em: https://population.un.org/wup/Publications/Files/WUP2018-Highlights.pdf . Acesso em: 03 fev. 2021.

[2] MOROZOV, E.; BRIA, F. A Cidade inteligente - Tecnologias urbanas e democracia. 1. ed. São Paulo: Ubu Editora, 2020.

[3] ALMEIDA, F. A. D. S. Modelando a informação da cidade: do estado da arte à construção de um conceito de City Information Modeling (CIM). 2018. Dissertação (Mestrado em Desenvolvimento Urbano) - Universidade Federal de Pernambuco, Recife, Pernambuco, 2018.

[4] ABRUCIO, F. L.; FRANZESE, C. Federalismo e Políticas Públicas: O Impacto das Relações Intergovernamentais no Brasil. In: Maria Fátima Infante Araújo; Lígia Beira. (Org.). Tópicos de Economia Paulista para Gestores Públicos. 1ed.Edições FUNDAP: São Paulo, 2007 , v. 1 , p. $13-3$. 
[5] JAIME, I. D. S. AS CIDADES CONTEMPORÂNEAS E SUAS TECNOLOGIAS: A PERSPECTIVA DO CITY INFORMATION MODELING. 2019, Dissertação (Mestrado em Projeto e Cidade) - Universidade Federal de Goiás, Goiania, Goias, 2019.

[6] PEREIRA, A. P. OS DESAFIOS PARA A IMPLEMENTAÇÃO DO CITY INFORMATION MODELLING COMO INSTRUMENTO NA GESTÃO URBANA: O CASO DE CURITIBA, PARANÁ. 2018, Dissertação (Mestrado em Gestão Urbana) - Pontifícia Universidade Católica do Paraná, Curitiba, Paraná, 2018.

[7] OLIVEIRA, F. M. DESENVOLVIMENTO DE FERRAMENTA BIM PARA AVALIAÇÃO PRESCRITIVA DE EFICIÊNCIA ENERGÉTICA INTEGRADA AO PROCESSO DE PROJETO. 2019, Tese (Doutorado em Dinâmicas do Espaço Habitado) - Universidade Federal de Alagoas, Maceió, Alagoas, 2019.

[8] Lacerda, D. P. Dresch, A. Proença, A. and. Antunes Júnior, J. A. V. Design Science Research: Método de pesquisa para a engenharia de produção, Gestão e Produção., vol. 20, no. 4, pp. 741-761, 2013, doi: 10.1590/S0104-530X2013005000014.

[9] SABOYA, R. T. Concepção de um sistema de suporte à elaboração de planos diretores participativos. 2007, Tese (Doutorado em Engenharia Civil) - Universidade Federal de Santa Catarina, Florianópolis, Santa Catarina, 2007.

[10] SASS, S. R. R. et al. Aplicação de técnicas de descoberta de conhecimento em banco de dados cadastrais para auxiliar no processo de tomada de decisão. Revista Brasileira de Cartografia. v. 66, n. 6, 2014.

[11] MARVIN, S.; LUQUE-AYALA, A. Urban Operating Systems: Diagramming the City. International Journal of Urban and Regional Research. v. 41, n. 1, p. $84-103,2017$. DOI: https://doi.org/10.1111/1468-2427.12479,

[12] SILVA JÚNIOR, F. A. O uso de sistemas generativos como instrumento de desenho urbano sustentável. 2016, Tese (Doutorado em Arquitetura e Urbanismo) - Universidade de Brasília, 2016, Brasília, Distrito Federal. Disponível em: http://repositorio.unb.br/handle/10482/21577.

[13] AUTILI, M. et al. A choreography-based and collaborative road mobility system for L'Aquila city. Future Internet. v. 11, n. 6. 2019. DOI: https://doi.org/10.3390/fi11060132.

[14] JIN, Y.; LIANG, H.; LI, Y. Spatial database of traffic management design and implementation. Information Technology Journal. v. 12, n. 22, p. 6909-6915. 2013. DOI: https://doi.org/10.3923/ITJ.2013.6909.6915.

[15] LOCK, O.; BEDNARZ, T.; PETTIT, C. The visual analytics of big, open public transport dataa framework and pipeline for monitoring system performance in Greater Sydney. Big

Earth Data. v. 5, n. 1, p. 134-159, 2020. DOI: https://doi.org/10.1080/20964471.2020.1758537

[16] GOLINI, R. et al. An assessment framework to support collective decision making on urban freight transport. Transport. v. 33, n. 4, p. 890-901, 2018. DOI: https://doi.org/10.3846/transport.2018.6591

[17] KHAN, Z. et al. An architecture for integrated intelligence in urban management using cloud computing. Journal of Cloud Computing. v. 1, n. 1, p. 1-14. 2012. DOI: https://doi.org/10.1186/2192-113X-1-1

[18] SOUZA, A. et al. Using Big Data and Real-Time Analytics to Support Smart City Initiatives. IFAC-PapersOnLine. v. 49, n. 30, p. 257-262, 2016. DOI: https://doi.org/10.1016/j.ifacol.2016.11.121

[19] ACUTO, M. et al. Informing urban governance? Boundary-spanning organizations and the ecosystem of urban data. Area. v. 51, n. 1, p. 94-103, 2019.DOI: https://doi.org/10.1111/area.12430 
[20] SHOJAEI, D. et al. Design and development of a 3D digital cadastre visualization prototype. ISPRS International Journal of Geo-Information. v. 7, n. 10, 2018. DOI: https://doi.org/10.3390/ijgi7100384

[21] ALMEIDA, A. C. L. Multi actor multi criteria analysis (MAMCA) as a tool to build indicators and localize sustainable development goal 11 in Brazilian municipalities. Heliyon. v. 5, n. 8, 2019. DOI: https://doi.org/10.1016/j.heliyon.2019.e02128. 